\title{
Single-nucleus chromatin accessibility reveals intratumoral epigenetic heterogeneity in IDH1 mutant gliomas
}

\author{
Ruslan Al-Ali ${ }^{1,2}$, Katharina Bauer ${ }^{3}$, Jong-Whi Park' ${ }^{1}$ Ruba Al Abdulla ${ }^{4}$, Valentina Fermi ${ }^{5}$, Andreas von Deimling ${ }^{6,7}$, \\ Christel Herold-Mende ${ }^{5}$, Jan-Philipp Mallm³,8, Carl Herrmann" ${ }^{3}$, Wolfgang Wick ${ }^{1,10}$ and Şevin Turcan ${ }^{1 *}$ (D
}

\begin{abstract}
The presence of genome-wide DNA hypermethylation is a hallmark of lower grade gliomas (LGG) with isocitrate dehydrogenase (IDH) mutations. Further molecular classification of IDH mutant gliomas is defined by the presence (IDHmut-codel) or absence (IDHmut-noncodel) of hemizygous codeletion of chromosome arms 1p and 19q. Despite the DNA hypermethylation seen in bulk tumors, intra-tumoral heterogeneity at the epigenetic level has not been thoroughly analyzed. To address this question, we performed the first epigenetic profiling of single cells in a cohort of 5 gliomas with IDH1 mutation using single nucleus Assay for Transposase-Accessible Chromatin with high-throughput sequencing (snATAC-seq). Using the Fluidigm HT IFC microfluidics platform, we generated chromatin accessibility maps from 336 individual nuclei, and identified variable promoter accessibility of non-coding RNAs in LGGs. Interestingly, local chromatin structures of several non-coding RNAs are significant factors that contribute to heterogeneity, and show increased promoter accessibility in IDHmut-noncodel samples. As an example for clinical significance of this result, we identify CYTOR as a poor prognosis factor in gliomas with IDH mutation. Open chromatin assay points to differential accessibility of non-coding RNAs as an important source of epigenetic heterogeneity within individual tumors and between molecular subgroups. Rare populations of nuclei that resemble either IDH mutant molecular group co-exist within IDHmut-noncodel and IDHmut-codel groups, and along with non-coding RNAs may be an important issue to consider for future studies, as they may help guide predict treatment response and relapse.

A web-based explorer for the data is available at shiny.turcanlab.org.
\end{abstract}

\section{Introduction}

Isocitrate dehydrogenase 1 (IDH1), and to a lesser extent, its mitochondrial homolog, IDH2, are mutated in a majority of adult lower grade gliomas (LGGs) [46]. IDH proteins normally serve as the core metabolic enzymes in the citric acid cycle and convert isocitrate to $\alpha$-ketoglutarate $(\alpha \mathrm{KG})$. The most common IDH1 mutation in gliomas (IDH1 R132) occurs in the catalytic domain of IDH1 and confers the ability to produce 2hydroxyglutarate (2-HG) [7]. 2-HG competitively inhibits enzymes that use $\alpha \mathrm{KG}$ as a cofactor, such as TET family of enzymes and Jmj-C domain containing histone demethylases, leading to DNA hypermethylation,

\footnotetext{
* Correspondence: sevin.turcan@med.uni-heidelberg.de

${ }^{1}$ Neurology Clinic and National Center for Tumor Diseases, University

Hospital Heidelberg, INF 460, 69120 Heidelberg, Germany

Full list of author information is available at the end of the article
}

and aberrant methylation of a number of histone marks along with impaired differentiation leading to an expansion of stem/progenitor cells [10, 27, 40]. Gliomas with IDH mutation exhibit global DNA hypermethylation and are subdivided into two distinct molecular subgroups: IDHmut-codel (hemizygous co-deletion of chromosome arms 1p/19q) and IDHmut-noncodel (without codeletion of $1 \mathrm{p} / 19 \mathrm{q}$ ) gliomas [4]. While transcriptional heterogeneity at the single cell level and longitudinal alterations in the bulk epigenomes of IDH mutant gliomas have been investigated, little is known about intratumoral epigenetic heterogeneity at the single cell level $[16,39,43]$. To address this question, we interrogated the accessible chromatin at the individual cell level in gliomas with IDH mutation using single nucleus Assay for Transposase-Accessible Chromatin with high-throughput

(c) The Author(s). 2019 Open Access This article is distributed under the terms of the Creative Commons Attribution 4.0 International License (http://creativecommons.org/licenses/by/4.0/), which permits unrestricted use, distribution, and reproduction in any medium, provided you give appropriate credit to the original author(s) and the source, provide a link to the Creative Commons license, and indicate if changes were made. The Creative Commons Public Domain Dedication waiver (http://creativecommons.org/publicdomain/zero/1.0/) applies to the data made available in this article, unless otherwise stated. 
sequencing (snATAC-seq) on a subset of 5 patient samples.

Using the Fluidigm microfluidics platform, we established a biologically-relevant analysis to overcome the technical limitation and high background noise associated with snATAC-seq. We identified heterogeneity in promoter accessibility within and between IDHmutcodel and IDHmut-noncodel samples. Interestingly, our results indicate differential accessibility of non-coding RNAs such as the CYTOR locus that exhibits a profound increase in promoter accessibility within IDHmutnoncodel tumors. Furthermore, we identify CYTOR as a poor prognosis factor in gliomas with IDH mutation. Overall, our results point to differential accessibility of non-coding RNAs as an important source of epigenetic heterogeneity within individual tumors and between molecular subgroups. The molecules identified are promising targets for future molecular research.

\section{Results}

Our cohort was primarily composed of WHO grade II gliomas, with the exception of one WHO grade III IDHmut-noncodel glioma, and all tumors harbored an IDH1 R132 mutation (Table 1). We used the Fluidigm HT IFC microfluids platform to perform snATAC-seq. We started with 7 tumors samples from glioma patients, but only 5 samples passed the quality control. We used stringent cut-offs and excluded unreliable cells, obtaining DNA accessibility maps for a total of 336 cells (Additional file 1: Table S1). Our analysis indicated a previously undescribed presence of identical reads leaking across specific rows and columns of the microfluidic chamber (Additional file 2: Figure S1a, b). We reasoned that these crosscontaminating reads were unlikely to be biologically relevant and may indicate an inherent technical issue with the microfluidics platform. To overcome this technical noise, we removed the leaky reads from all samples (Additional file 2: Figure S1c-f). Overall, we obtained high-quality DNA accessibility maps from 145 cells from 3 IDHmut-noncodel gliomas (Astro1, Astro2, Astro3), and 191 cells from 2 IDHmut-codel gliomas (Oligo1, Oligo2), with a total of 336 cells (Additional file 1: Table S1).

We applied a pipeline (HOMER), commonly used for peak calling from ChIP-seq data, to call peaks in our snATAC-seq datasets on both pseudo-bulk and single

Table 1 Patient samples used for snATAC-seq

\begin{tabular}{lllll}
\hline Pseudonym & IDH mutation & Histology & Grade & Sample ID \\
\hline NCH5526 & IDH1 R132S & Astrocytoma & Grade II & Astro1 \\
NCH6015 & IDH1 R132H & Astrocytoma & Grade II & Astro2 \\
NCH5559 & IDH1 R132H & Astrocytoma & Grade III & Astro3 \\
NCH5540 & IDH1 R132H & Oligodendroglioma & Grade II & Oligo1 \\
NCH5699 & IDH1 R132H & Oligodendroglioma & Grade II & Oligo2 \\
\hline
\end{tabular}

nuclei level. Peaks called from pseudo-bulk profiles of the snATAC-seq data closely resembled bulk ATAC-seq data obtained from IDH mutant TCGA LGG samples (Fig. 1a) [6]. For all samples, majority of the reads centered around the transcription start sites (TSS) (Fig. 1b). Reads were distributed evenly and proportionally across chromosomes (Fig. 1c). The majority of peaks mapped to intronic and enhancer regions (Fig. 1d). We used the GREAT toolbox to assess whether the enhancer regions were enriched for any particular gene sets. Our analysis indicated that enhancers in IDHmut-noncodel samples were enriched in gene sets associated with suppression of pro-B cell differentiation and development. IDHmutcodel enhancer regions were enriched for gene sets associated with mRNA regulation, spinal cord oligodendrocyte cell fate and inhibition of neuroepithelial differentiation (Fig. 1e).

Next, to reduce noise, we decided to limit our analysis to accessible peaks identified from bulk LGGs. To achieve this, we overlapped the peaks from our snATAC-seq data with the peaks called from bulk ATAC-seq of TCGA LGG samples [6] (Additional file 2: Figures S2 and S3). We used these overlapping peaks for the remainder of our study. Subsequently, we applied t-distributed stochastic neighbor embedding (t-SNE) to reduce the dimensionality of the snATAC-seq data. The t-SNE mapping showed three clusters, branched along two trajectories (upper and lower), coalescing on two clusters as indicated with red and blue circles (Fig. 2a). Approximately $20 \%$ of the cells could not be classified and remained in a gray zone due to lack of sufficiently specific peaks, while the upper and lower clusters were distinct, and almost entirely consisted of cells from a particular molecular subtype (Fig. 2a). Heatmap of open promoters unique to each trajectory indicates variable accessibility within or between IDHmutcodel or IDHmut-noncodel samples (Fig. 2b).

To determine whether $1 \mathrm{p} / 19 \mathrm{q}$ codeletion can be detected from the snATAC-seq data, we inferred largescale copy number alterations in these chromosomes by averaging ATAC-seq coverage for each nuclei. This analysis indicated decreased coverage for both $1 \mathrm{p}$ and $19 \mathrm{q}$ arms relative to 1q and 19p in IDHmut-codel tumors, revealing molecular evidence in the snATAC-seq data for the codeletion pattern in these samples (Fig. 2c).

\section{Differential accessibility of transcription factors}

To determine whether chromatin accessibility within transcription factor (TF) binding sites differ, we applied chromVAR to identify highly variable TF motifs [33] (Additional file 3: Table S2, Additional file 2: Figure S4a). We used the TF z-scores to visualize the snATAC-seq data using t-SNE. Similar to Fig. 2a, these features also separated the data into two states, driven by differences in molecular subtype (Fig. 2d). A heatmap visualization 


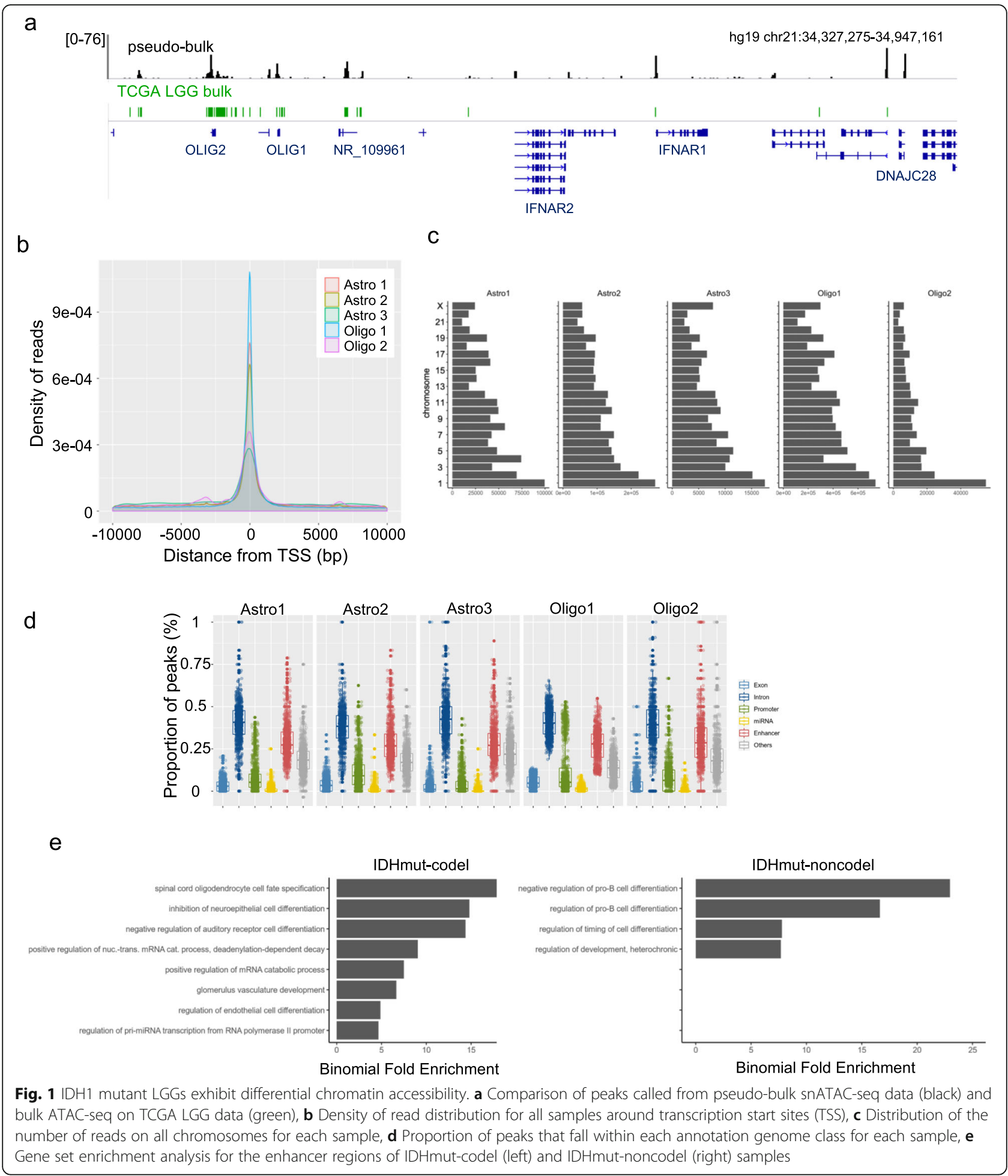

showed heterogeneity of TF accessibility within samples belonging to a specific molecular subgroup (Additional file 2: Figure S4b), We identified 14 TFs that exhibited high variability $(>1.5)$ (Fig. 2e). The highest variability was observed by differences in two major families of AP-1 transcription factors: JUN and FOS that are expressed downstream of the mitogen-activated protein kinase (MAPK) signaling cascades (Fig. 2f). A subgroup of IDHmut-codel samples also displayed differential NHLH1 accessibility (Fig. 2g). NHLH1 is required for the formation of pre-cerebellar neurons in the hindbrain [34]. In addition, PAX5 showed differential accessibility in 


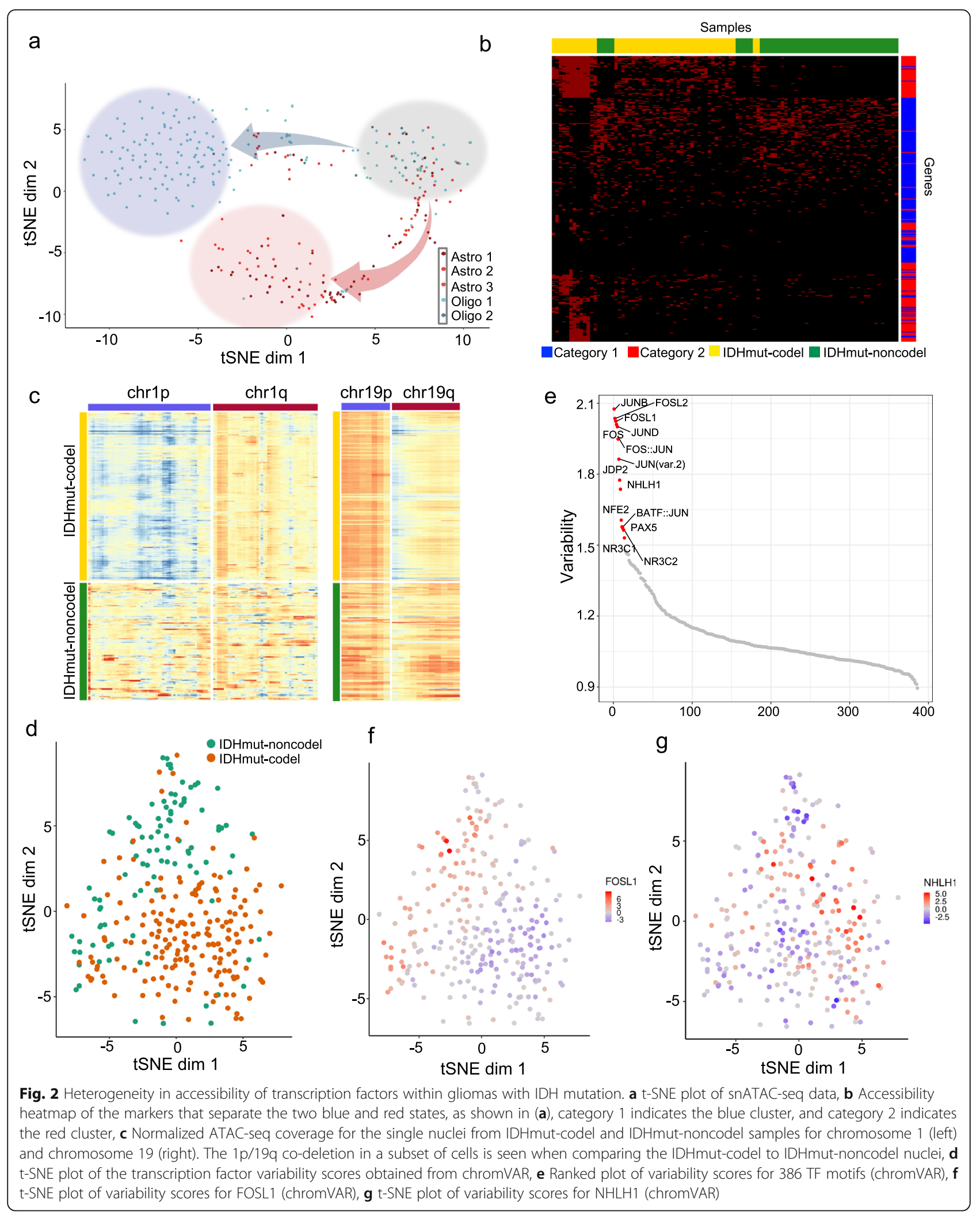


a subset of cells (Additional file 3: Table S2). PAX5 plays an essential role for normal development of the midbrain and cerebellum [41]. Moreover, several known oncogenic transcription factors, such as the ETS family, that are aberrantly activated in a majority of IDHmut-codel tumors, also exhibited significant variability within IDHmut-codel and IDHmut-noncodel tumors [19].

\section{Non-coding RNAs and transcription factors are differentially accessible in gliomas with IDH mutation} We identified IDHmut-noncodel and IDHmut-codel specific peaks near the promoters of several non-coding RNAs. These included LINC01193, CCT8L2 and MIR4436A, that exhibited increased chromatin accessibility in IDHmutnoncodel samples, and MALAT1 with increased chromatin accessibility in IDHmut-codel samples (Fig. 3a). MALAT1 and LINC01193 are known to have functions in cancer $[12,47]$. We also identified accessible promoters enriched in IDHmut-codel samples, including OLIG2, and KLF12 (Fig. 3a, Additional file 4: Table S3). Of note, OLIG2, an essential transcription factor for inducing oligodendrocyte development, has an open promoter in the majority of IDHmut-codel nuclei, whereas only a few nuclei in IDHmut-noncodel samples harbor an accessible OLIG2 promoter (Fig. 3a).

\section{CYTOR as an example for differentially enriched non- coding RNA with clinical significance}

Our snATAC-seq data pointed to a striking difference in accessibility around and within the CYTOR promoter. The t-SNE visualization revealed accessible CYTOR promoter along the lower branch trajectory, which is largely defined by IDHmut-noncodel samples (Fig. 3b). Specifically, 70\% (101/145) of IDHmut-noncodel samples, and $10.5 \%(20 / 191)$ of IDHmut-codel samples had an open CYTOR promoter (Fig. 3c). We searched for co-accessible regions with the CYTOR promoter in the combined snATAC-seq dataset and identified 50 correlated regions (Pearson > 0.2) (Fig. 3d, Additional file 5: Table S4). Interestingly, one of the correlated regions was the promoter of ID2, a transcriptional regulator that supports a prosurvival role in malignant gliomas by inactivating VHL $[22,48]$. CYTOR (LINC00152) is a long non-coding RNA that regulates cytoskeleton and plays an oncogenic role in several cancers, including colorectal cancer, and gastric cancer $[45,49]$. CYTOR is associated with poor prognosis and is upregulated in diffuse gliomas, and higher grade IDH wild-type gliomas and glioblastomas [31, 52].

To assess the methylation state of the CYTOR locus, we utilized data from 68 gliomas with IDH mutation profiled using the Illumina Infinium MethylationEPIC BeadChip (EPIC) arrays as a part of the DNA-methylation based classification efforts of central nervous system tumor entities [5]. The methylation data included samples from 31 IDHmut-noncodel and 37 IDHmut-codel. We identified 10,634 differentially methylated sites between the two IDH molecular subgroups at a q-value $<0.001$ and absolute $\beta$-value $>0.1$ (Additional file 6: Table S5). To determine whether there was an inverse correlation between accessible chromatin and DNA methylation upstream of CYTOR TSS, we overlapped the location of the EPIC array probes with the snATAC-seq peaks called from pseudo-bulk regions. This analysis identified two overlapping CpG probes: cg22535363, and cg23944790 (Fig. 3e). Both of these probes were significantly hypermethylated in IDHmut-codel samples when compared to IDHmut-noncodel samples (Fig. 3f), further suggesting the limited chromatin accessibility of CYTOR in IDHmutcodel gliomas.

Next, we wondered whether these differences in CYTOR at the DNA level were reflected at a transcriptional level. To answer this question, we analyzed bulk RNA-seq data from the Chinese Glioma Genome Atlas (CGGA), which includes two datasets with 693 (CGGA1) and 325 (CGGA-2) samples, respectively. We restricted our analyses to IDH mutant gliomas (WHO Grade II-IV) within the CGGA data, which included 258 CGGA-1 samples and 152 CGGA-2 samples. CYTOR expression was significantly higher in IDHmut-noncodel samples compared to IDHmut-codel samples in both datasets (Fig. 4a). Overlap of genes positively correlated with CYTOR in both datasets (198 genes in CGGA-1, and 541 genes in CGGA-2) (Pearson > 0.5) revealed 101 genes with high correlation to $C Y T O R$ (Additional file 7 : Table S6) Interestingly, these genes were significantly enriched for several pathways including regulation of migration, regulation of vasculature development, and collagen formation (Fig. 4b, Additional file 8: Table S7). Finally, we asked whether CYTOR expression was correlated with overall survival in gliomas with IDH mutation (WHO Grade II-III). We identified the maximal cut-off point and classified each tumor by high- or low- CYTOR expression. Comparison of IDHmut-noncodel and IDHmut-codel samples revealed that high CYTOR expression was associated with poor overall survival prognosis in both CGGA and TCGA data sets (Fig. 4c, d, Additional file 2: Figure S6a, b).

\section{Discussion}

Gliomas with IDH mutation exhibit global DNA hypermethylation. While previous studies have investigated heterogeneity at the transcriptional level, it is unclear whether these tumors harbor epigenetic heterogeneity at the individual cell level. To our knowledge, our study is the first description of chromatin accessibility of gliomas with IDH mutation using single nucleus ATAC-seq. Using conservative cutoff values, and by relying on overlapping peaks from bulk ATAC-seq from IDH mutant 


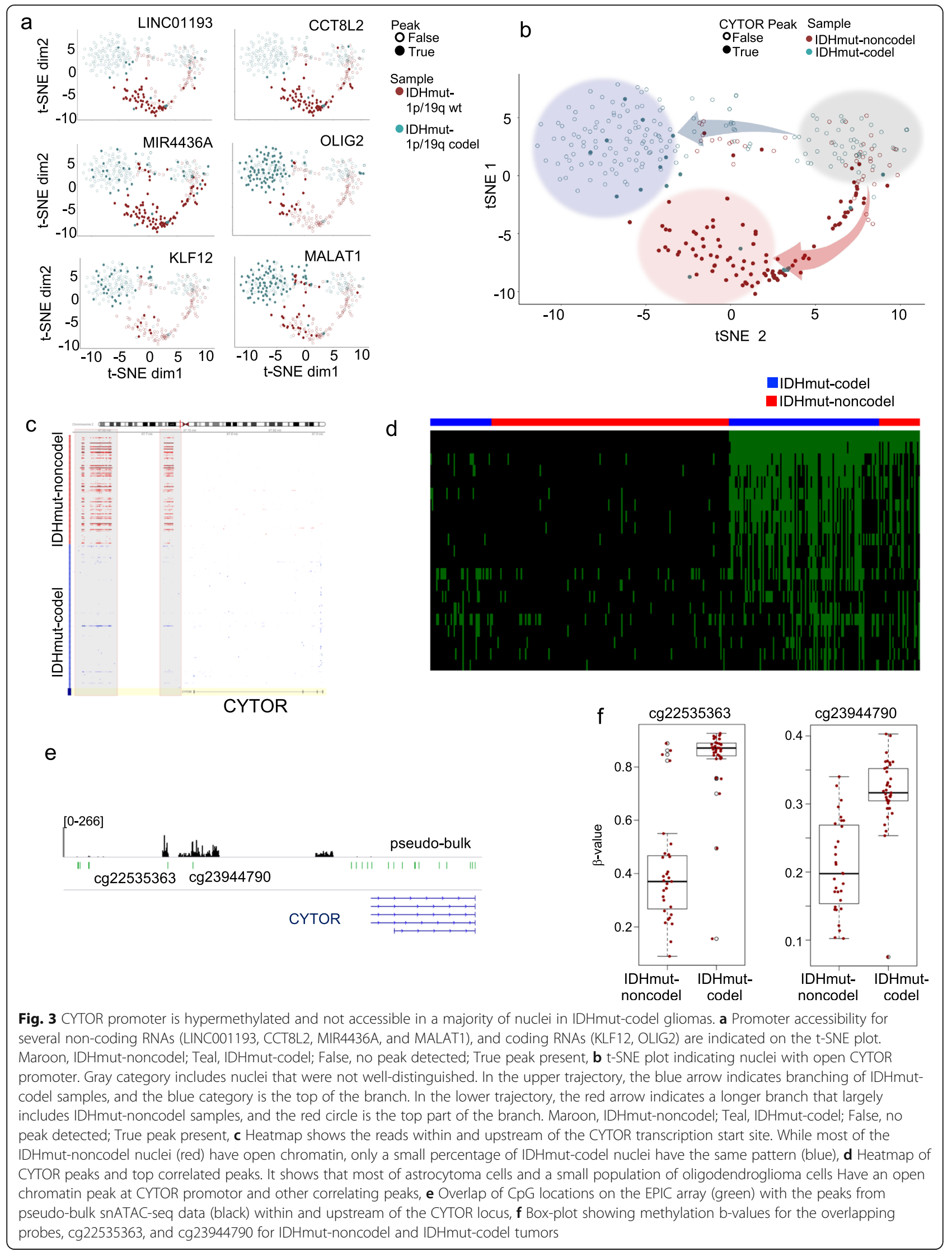




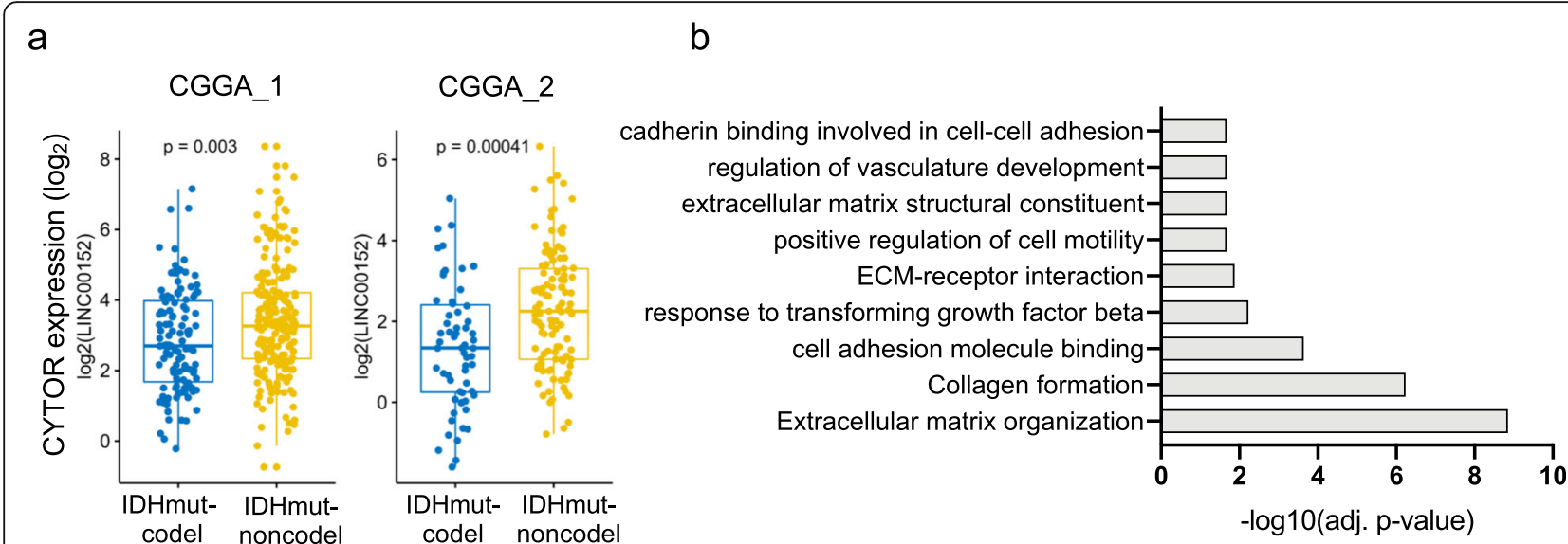

C

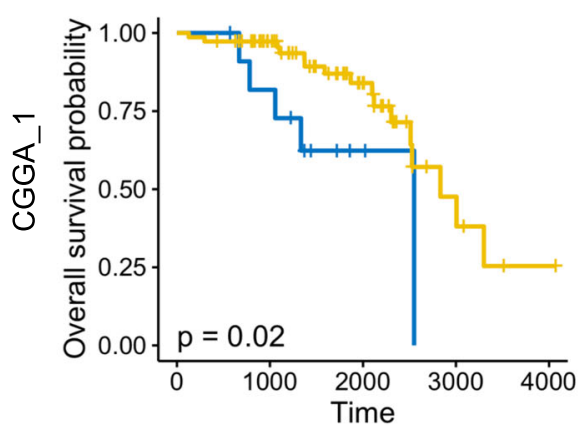

d

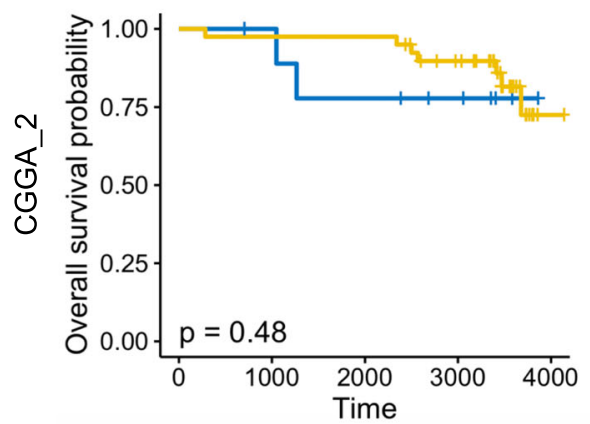

IDHmut-noncodel
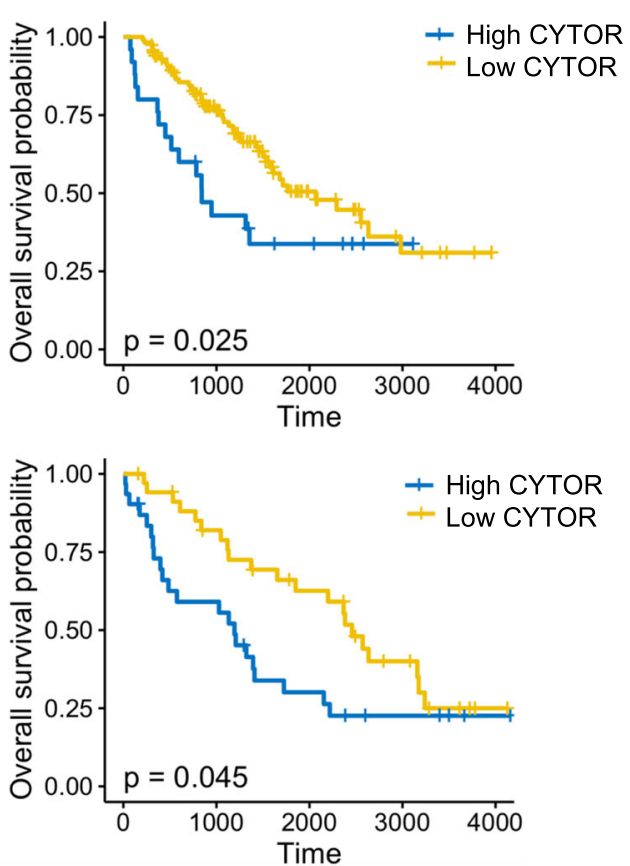

Fig. 4 CYTOR is associated with poor overall survival in gliomas with IDH mutation. a Boxplot indicates CYTOR expression in IDHmut-codel (blue) and IDHmut-noncodel samples (yellow) in the Chinese Glioma Genome Atlas (CGGA_1, and CGGA_2), b Functional enrichment of genes

correlating with CYTOR in both CGGA cohorts. Adj. p-value, adjusted p-value, c Kaplan-Meier plots for IDHmut-codel (left), and IDHmut-noncodel (right) gliomas indicating survival between samples with high and low CYTOR expression in CGGA_1 dataset, d Kaplan-Meier plots for IDHmutcodel (left), and IDHmut-noncodel (right) gliomas indicating survival between samples with high and low CYTOR expression in CGGA_2 dataset

glioma patients samples, we were able to reduce noise and constrain our analysis to biologically significant regions. We observed differential accessibility of several transcription factor binding sites, within and between IDHmut-codel and IDHmut-noncodel samples. Accessibility of DNA binding sites for JUN or FOS were highly variable, and interestingly were accessible to a much lower extent in IDHmut-codel tumors. Activity of AP-1, the transcriptional activator composed of members of the Jun and Fos families are regulated by MAPK signaling, and control proliferation and apoptosis [35].
Sequence binding-specific sites of AP-1 transcription factors are reported to be hypomethylated targets in glioblastoma with poor clinical outcome and low glioma CpG Island Methylator Phenotype (G-CIMP) [8]. Several transcription factors, such as NHLH1 and PAX5 with known roles in neurodevelopment also exhibit differential accessibility among IDHmut-codel and IDHmut-noncodel samples. Although this remains to be determined, it is possible that these accessible TF motifs are vestiges of cell of origin for these tumors. This is also suggested by gene set enrichment analysis which showed significant enrichment 
for neural lineage differentiation pathways, particularly for IDHmut-codel gliomas. In addition, we identify that ETS transcription factors display heterogeneity with increased variability in accessibility in IDHmut-noncodel tumors. The ETS/AP-1 transcription factors regulate a RAS-responsive gene expression program. PEA3 subfamily of ETS proteins (ETV1, ETV4, ETV5) amplify transcriptional signals when RAS/MAPK signaling pathway is active, and abolishing Ets activity leads to a block in glioma initiation $[2,14]$.

Our data show that majority of IDHmut-codel nuclei, and only a subset of IDHmut-noncodel nuclei harbor an open chromatin within the promoter of OLIG2. Expression of OLIG2 is restricted to the central nervous system and determines oligodendrocyte and astrocyte fate determination in the developing brain $[28,50,51]$. It is ubiquitously expressed in gliomas, and was identified as one of the core transcription factors that can reprogram differentiated GBM cells into glioma stem cells [25, 26, 37]. Our results indicate that CYTOR could be of importance in the pathogenesis of IDH mutant tumors, as high CYTOR expression is associated with poor overall survival. Given our small sample size, we were unable to determine whether chromatin accessibility of CYTOR varied by grade in IDHmut-codel samples. However, our integrative data indicates that a subset of IDHmut-codel tumors also harbors an open chromatin for CYTOR and having an open promoter might be indicative of a malignant subpopulation associated with progression within Grade II IDHmut-codel gliomas. Therefore, it may be possible to target such existing programs at an early stage of tumor development, that could offer a therapeutic benefit for patients. However, future studies are needed to elucidate such interactions during malignant progression.

A limitation of scATAC-seq studies is due to sparsity of data, it is challenging to determine cell identity. Given that IDHmut-codel samples harbor chromosome arm deletions, we were able to infer the presence of $1 \mathrm{p} / 19 \mathrm{q}$ deletions in a large majority of our snATAC-seq data. In addition, tumor-associated microglia and macrophages (TAMs) are the most abundant cell types that infiltrate gliomas [9, 43]. However, we did not observe peaks within the promoter for several well-described TAM markers, such as CD11b and CX3CR1, IBA1 or CD45 (data not shown) [1]. Taken together, these results suggest that a large proportion of the signal is obtained from malignant tumor cells.

\section{Materials and methods}

\section{Patients and tumors}

All tumors were obtained from patients following surgical resection at the Department of Neurosurgery at the University Hospital Heidelberg, Germany. Use of patient material was approved by the Institutional Review Board at the Medical Faculty of Heidelberg.
Informed consent was obtained from all patients included in the study. Each sample was examined histologically for sufficient tumor cell content of at least $60 \%$ and diagnosed by a neuropathologist. The pathologic characteristics of the tumor samples are summarized in Additional file 1: Table S1. RNA-seq data obtained from 693/325 Grade II-IV and 514 Grade IIIII glioma samples were downloaded from the Chinese Glioma Genome Atlas (CGGA) and the Cancer Genome Atlas (TCGA), respectively, along with their corresponding clinical information.

\section{Sample preparation}

Following surgical resection, fresh tumor samples were separated into single cell suspensions via mechanical and collagenase enzymatic dissociation (StemCell Technologies- Canada). Debris was removed using tubes with strainer cap (Corning, USA-NY). The cells were snap frozen in a stem cell freezing media (CTS Synth-aFreeze Medium, Life Technologies) and stored in liquid nitrogen until diagnosis was confirmed.

\section{Sequencing and quality control}

Nuclei were obtained using Nuclei EZ Prep (SigmaAldrich, USA-MO). The single nucleus ATAC-Seq protocol performed on HT IFC microfluidics system (Fluidigm, USA-CA) and HiSeq 2000 sequencing technologies (Illumina, USA-CA) with an adapted protocol (Additional file 2: Figure S5). Nuclei were incubated with Tn5 off-chip. The reaction was stopped with EDTA and the nuclei are loaded on the C1 HT IFC. During the amplification in the IFC the first barcode was added to the library which allows pooling per column. After harvesting the PCR products were purified with Ampure beads at a $1.4 \mathrm{x}$ ratio. The second barcode was then introduced by a second PCR off-chip and the libraries were size selected with AMPure beads (Beckman Coulter, Brea, CA). Each pool was subjected to quality control using Qubit (ThermoFischer, USA-MA) and TapeStation D1000 (Agilent, USA-CA).

Overall, we tested samples from 7 patient samples, however only 5 samples provided material with sufficient quality for sequencing. One oligodendroglioma sample (NCH5540) was sequenced with 75 bp single reads, while the rest of the samples were sequenced with $50 \mathrm{bp}$ single reads. Duplicate nuclei in C1-800 chip were estimated with visual microscopic analysis, and quality control (QC) was checked at each step. We tested 3 IDHmutnoncodel and 2 IDHmut-codel patient samples. After data processing, 145 cells from IDHmut-noncodel, and 191 cells from IDHmut-codel samples passed our cutoffs with selective peak-calling. 


\section{Computational analysis of snATAC-seq data}

\section{Data clean-up and alignment}

Our initial analysis revealed that some cells have exactly the same read sequences; a phenomenon that cannot be explained by Tn5 enzyme-selectivity alone. The topological spread of the clustered cells revealed that they are mostly located along the same row on the chip, and to a lesser extent along the same columns (Additional file 2: Figure S6-d). These distributed reads are unlikely to originate from individual cells, and potentially indicate to an imperfection within the microfluidics platform. We hypothesize that some wells with a high number of reads spill over to neighboring wells along the microfluidic platform. Target wells are probably empty as they have low number of unique reads with less variability. One explanation for the event might be that wells with duplicated nuclei generate irregular fluid movement that causes asynchronized nuclei lysis or major concentration gradient and differences in liquid densities. This is supported by the fact that the empty chambers are the main target to be filled with the leaking reads. A large part of our analysis focused on cleaning the data and performing quality controls. To handle the well-to-well leakage, we applied the standard de-duplication with Picard's MarkDuplicate (http:// broadinstitute.github.io/picard) but failed to reduce biases due to leakages. We have chosen to remove all reads that exist in more than one well before alignment using custom python3.7 script (described later). Noticeably, fastq files were inflated with PCR amplicons and some read sequences sustained mutations after PCR amplification and sequencing. A small percentage in total, this kept introducing bias to the final result. Ultimately, we deduplicated fastq files using clumpify (sourceforge.net/projects/bbmap/) with default dedupe parameters which removed most of the duplicates and the mutant amplicons. Then, we applied the custom python3.7 script to remove the leaky reads among the cells. The script reads each individual fastq file, iterates through each of the other files in the sample/HT-IFC chip, then removes any duplicated read. The script removed the exact sequence of DNA that exist in two or more wells. Remaining mutant sequences were not addressed due to unreasonable computational costs. The amount of leakages is reduced by $98 \%$ (Additional file 2: Figure S6). As expected, reads tend to gather around transcription start site (TSS) (Additional file 2: Figure S1-b). Every analysis that was performed later is done on these cleaned data. De-duplicated files were aligned with bowtie2 [20] with default parameters against GRCh37.p13 (GenCode), sorted, non-standard chromosomes were removed and bam files were indexed using Samtools 1.9 [23]. Finally, the wells were checked for leaky reads in bed files: percentage of reads with the same coordinates were measured with and without the python script (Additional file 2: Figure S5-b).

\section{Bioinformatic analysis}

To reduce bias, we called peaks in single cells and used published bulk ATAC-Seq for LGG from TCGA as reference [6]. We called peaks with HOMER v4.10 [13], applying makeTagDirectory with keepAll option. Then we used findPeaks with style histone, minDist 10,000 and size 10,000 . The peaks were converted to bed format using pos2bed.pl (HOMER) and annotated with annotatePeaks.pl (HOMER) for hg19. We overlapped our snATAC-Seq peaks with the reference peaks. We defined a peak to have was considered when the overlap is at least $50 \mathrm{bp}$. Peaks with less than 5 cells were excluded, then cells with less than 50 positive peaks. To remove duplicated nuclei, we removed top $5 \%$ of the cells by selecting off the cells with more than 3000 peaks. After applying those parameters, we obtained 336 cells and peaks for 4609 genes. Cells were clustered with t-SNE test for 2 dimensions with perplexity $=30$ using Rtsne v0.15 [42] with $\mathrm{R}$ v3.6.1. We analyzed read distance from Transcription Starting Sites (TSS). The distance forms a peak of high density in all samples, and some minor peaks further (Fig. 1b). We used TSS table based on atacR v0.4.14 [36], and compared the reads from all wells of a psuedo-bulk file using Samtools 1.9, Rsamtools [30] and GenomicRanges 1.36.0 [21]. Reads around TSS in a range of $2 \mathrm{~kb}(-2000$ to +2000$)$, are 11.4, $8.8,5.8,17.2$ and $6.6 \%$ for samples astro1, astro2, astro3, oligo 1 and oligo 2 respectively.

\section{Enrichment}

Most of the peaks fall into intronic and intergenic annotation. We overlapped the intergenic peaks with miRNA data from USCS and with enhancers from HACER db [44]. The regions from enhancers were reduced using GenomicRanges and overlapped with Homer peaks from each cell. The intergenic peaks that were not classified as miRNA or enhancers were called "others" (Fig. 1d). We evaluated functional cis-regulatory regions for enhancers using Genomic Regions Enrichment of Annotation Tools (GREAT) [29]. We divided the cells into Oligodendrogliomas and Astrocytomas, and we obtained the enhancers that overlapped more than 10 cells. We generated a BED file from the peaks of each group, and we uploaded the file to GREAT website. We used gene ontology for Biological processes with over 4 Binomial Fold Enrichment.

\section{Clustering}

To identify clusters in our data and to find top expressed genes, we used $\mathrm{k}$-means algorithm $(k=5)$ in $\mathrm{R}$ v3.61 
base. The clusters were not perfect, so we improved manually the borders of the clusters (Additional file 2: Figure S3). The two branches consist of two clusters each, and the undefined nuclei cluster in the fifth.

Based on the manual clustering, we looked for top expressed genes and top specific genes for each group and each cluster. The equations for those two markers as follows:

$$
\text { TopGenes }=\operatorname{sort}\left(\mathrm{x}^{+} / \mathrm{Y}^{+}-(\mathrm{x}-+1) / \mathrm{Y}^{-}\right), \text {where } \mathrm{x}^{+}>10
$$

SpecificGenes $=\operatorname{sort}\left(\mathrm{x}^{+} / \mathrm{Y}^{+} /(\mathrm{x}-+1) / \mathrm{Y}^{-}\right)$, where $\mathrm{x}^{+}>10$

$\mathrm{x}^{+}$: Number of nuclei with peak(s) for the gene in the specific group/cluster

$\mathrm{Y}^{+}$: Total number of nuclei with peak(s) for the gene

$x-$ Number of nuclei with no peaks for the gene in the specific group/cluster

$\mathrm{Y}^{-}$: Total number of nuclei with no peaks for the gene

ATAC-seq coverage and CNV. We used a method similar to Satpathy et al. [32]. Chromosomes were tiled into $10 \mathrm{Mb}$ regions with $2 \mathrm{Mb}$ overlap (using the bedtools makewindows function), and the average ATAC-seq coverage for each cell was computed inside this window using the bigWigAverageOverBed function. Each window was normalized using 100 windows with matching GC content (excluding windows from the $1 p$ and $19 \mathrm{q}$ arm). The normalized coverage of a window $w_{O}$ was computed as

$$
\operatorname{CovNorm}\left(w_{0}\right)=\operatorname{mean}_{i=1 . .100} \log \left(\frac{\operatorname{cov}\left(w_{0}\right)+1}{\operatorname{cov}\left(w_{i}\right)+1}\right)
$$

were $w_{i}$ represent the GC-content matching windows. Telomeric and centromeric regions with low mapping percentage were manually excluded from the plots based on the mapping profiles over the single-cells.

\section{Transcription factor activity}

We used chromVAR v1.6.0 to infer transcription factor accessibility in our snATAC-seq. Counts were obtained with getCounts function using peaks from bulk ATACseq TCGA for LGG (peak-names start with LGG), a CG bias was corrected, and peaks filtered with min_depth = 500 and min_in_peaks $=0.013$. A total 280 cells passed the filtering process, which were matched to JasperMotifs [18] and genome from BSgenome.Hsapiens.UCSC.hg19 v1.4. Background peaks were prepared, and deviation was measured via computeDeviations function. Variability was calculated and plotted. deviationsTsne and plotDeviationsTsne were applied for the top ten genes. A heatmap for sample correlation was produced by the same tool with threshold of 1 (Additional file 2: Figure S4). The difference between number of cells that passed the
chromVAR criteria and those that passed our method can be attributed to the differences in the cutoff set by the method.

\section{Web-based explorer for data visualization}

We used the peaks we called and selected against bulk TCGA ATAC-Seq peaks as starting point to produce a web-based explorer of the data. Gene annotation were used from TCGA publication and all peaks related to one gene were collapsed after t-SNE clustering. A SHINY-R3.6 app (cran.r-project.org) was developed with ggplot2 library (H. Wickham. ggplot2: Elegant Graphics for Data Analysis. Springer-Verlag New York, 2016.) and tested on amazon cloud service (AWS-EC2) with 1GB of RAM and 1 core on Ubuntu 18 server instance, and a duplicate copy was tested on a similar server from hostinger.com to check platform compatibility. To visualize the data from selective peak-calling, we produced an explorer based on $\mathrm{R} /$ shiny package 1.3.2. The clustered data, the code and a manual are submitted at github.com/ RuslanAlali/SHINY_scATAC-Seq. Data can be visualized at shiny.turcanlab.org.

\section{Array-based methylation analysis}

Methylation analysis was performed using the Illumina HumanMethylationEPIC bead array at Heidelberg Neuropathology. R statistical software (v3.6.0) was used for data analysis. The minfi methylation pipeline was used to extract and analyze data from idat files, and normalization was carried out using functional normalization method [11]. Differentially methylated positions between IDHmutcodel and IDHmut-noncodel gliomas were detected using dmpFinder in minfi package. Loci with q-value $<0.001$ and absolute $\beta$-value $>0.1$ were considered to be differentially methylated.

\section{CGGA RNA-seq data analysis}

CGGA RNA-seq datasets were used to determine the Pearson's correlation coefficient for CYTOR versus all other genes. Genes with correlation coefficients $>0.5$ were considered to be positively correlated with CYTOR. To determine the functional enrichment of genes correlating with CYTOR, WebGestalt was used to perform overrepresentation using the following functional databases: Gene Ontology databases (molecular function, biological process), and Pathway (KEGG, and REACTOME) [24].

\section{Statistical analysis}

All statistical analyses were performed using the R software (v3.6.0). RNA-seq data from the CGGA cohort was downloaded, and processed in $\mathrm{R}$. The maxstat $\mathrm{R}$ package was used to determine the optimal cut-off points for CYTOR expression to dichotomize patients into low and high expression groups [15]. The survival and survminer 
$R$ packages were used for Kaplan-Meier analysis to estimate the survival curves of IDHmut-codel and IDHmutnoncodel subgroups $[17,38]$. Statistical comparison of groups was calculated using the log rank test. $P$-value less than 0.05 was considered statistically significant.

\section{Data availability}

Called peaks from Homer pipeline have been deposited in the Gene Expression Omnibus under accession number GSE137266.

\section{Conclusion}

In conclusion, although we obtained a limited number of nuclei from five samples, scATAC-seq is shown to be scalable to scRNA-seq level with thousands of cells [3]. By integrating the snATAC-seq data with unbiased data analysis from bulk methylation, RNA-seq and ATACseq datasets, we were able to provide initial insights into glioma epigenetics at the level of individual nuclei. As new high-throughput single cell ATAC-seq technologies have become available, we envision that future studies will further expand our understanding of the epigenetic order in gliomas.

\section{Supplementary information}

Supplementary information accompanies this paper at https://doi.org/10. 1186/s40478-019-0851-y.

\section{Additional file 1: Table S1. Statistics on SnATAC-seq data. \\ Additional file 2: Figure S1. The effect of removal of leaky reads from Oligo1. Figure S2. Different clustering approaches on snATAC-seq data. Figure S3. Cluster identification. Figure S4. chromVAR results on all 5 samples. Figure S5. Overview of snATAC workflow. Figure S6. Prognostic significance of CYTOR in IDH mutant gliomas.}

Additional file 3: Table S2. chromVAR analysis of snATAC-seq data indicates variability scores for 386 transcription factor motifs.

Additional file 4: Table S3. Genes that correlate with specific open chromatin in IDH mutant LGGs.

Additional file 5: Table S4. Regions correlating with CYTOR across all samples.

Additional file 6: Table S5. Differentially methylated probes between IDH-codel and IDH-intact samples (q-value $<0.001$ and absolute delta ßvalue $>0.1$.

Additional file 7: Table S6. Genes correlating with CYTOR in the CGGA cohorts.

Additional file 8: Table S7. Overrepresented gene sets in the 101 genes correlating with CYTOR.

\section{Acknowledgements}

We thank Kevin Jimenez-Cowell and the Single-cell open lab at DKFZ for helpful discussions. This work was supported by the German Cancer Aid (S.T.), epigenetics@dkfz program (S.T., C.H.), and DFG FOR2647 (J-P.M.).

\section{Authors' contributions}

ST and RA-Ali conceived the study. VF and CH-M provided pre-characterized tumor samples and clinical data. RA-Ali, KB prepared the single cell ATAC-seg experiments. J-PM developed and adapted the single cell ATAC-seq protocol for the Fludigim HT IFC microfluidic system. RA-Ali, and CH performed the bioinformatic analysis. RA-Ali and RA-Abdulla designed and set up the Shiny interface. ST and J-WP analyzed survival data. AvD contributed methylation data from tumors. $\mathrm{CH}$ and WW provided conceptual input and expertise. ST, and RA-Ali wrote the manuscript with input from all authors. All authors read and approved the final manuscript.

\section{Competing interests}

The authors declare that they have no competing interests.

\section{Author details}

${ }^{1}$ Neurology Clinic and National Center for Tumor Diseases, University Hospital Heidelberg, INF 460, 69120 Heidelberg, Germany. ${ }^{2}$ Institute Cochin، INSERM U1016, UMR 8104 CNRS, Faculté René Descartes, 24 rue du Faubourg St Jacques, 75014 Paris, France. ${ }^{3}$ Single-cell Open Lab, German Cancer Research Center (DKFZ), Heidelberg, Germany. ${ }^{4}$ Institute of Research, Development and Innovation in Healthcare Biotechnology in Elche- IDiBE, Elche, Spain. ${ }^{5}$ Division of Experimental Neurosurgery, Department of Neurosurgery, University of Heidelberg, INF 400, D-69120 Heidelberg, Germany. ${ }^{6}$ Department of Neuropathology, Institute of Pathology, University of Heidelberg, INF 224, D-69120 Heidelberg, Germany. ${ }^{7}$ Clinical Cooperation Unit Neuropathology, German Consortium for Translational Cancer Research (DKTK), German Cancer Research Center (DKFZ), Heidelberg, Germany. ${ }^{8}$ Division of Chromatin Networks, German Cancer Research Center (DKFZ) and BioQuant, Heidelberg, Germany. ${ }^{9}$ Health Data Science Unit, Medical Faculty University Heidelberg and BioQuant, 69120 Heidelberg, Germany. ${ }^{10}$ Clinical Cooperation Unit Neurooncology, German Cancer Consortium (DKTK), German Cancer Research Center (DKFZ), Heidelberg, Germany.

Received: 13 November 2019 Accepted: 18 November 2019 Published online: 05 December 2019

\section{References}

1. Bowman RL et al (2016) Macrophage ontogeny underlies differences in tumor-specific education in brain malignancies. Cell Rep 17(9):2445-2459

2. Breunig JJ et al (2015) Ets factors regulate neural stem cell depletion and Gliogenesis in Ras pathway Glioma. Cell Rep 12(2):258-271

3. Buenrostro JD et al (2015) Single-cell chromatin accessibility reveals principles of regulatory variation. Nature 523(7561):486-490

4. Cancer Genome Atlas Research Network et al (2015) Comprehensive, integrative genomic analysis of diffuse lower-grade Gliomas. N Engl J Med 372(26):2481-2498

5. Capper D et al (2018) DNA methylation-based classification of central nervous system tumours. Nature 555(7697):469-474

6. Corces MR et al (2018) The chromatin accessibility landscape of primary human cancers. Science. 362(6413).

7. Dang $L$ et al (2009) Cancer-associated IDH1 mutations produce 2hydroxyglutarate. Nature 462(7274):739-744

8. de Souza CF et al (2018) A distinct DNA methylation shift in a subset of Glioma CpG Island Methylator phenotypes during tumor recurrence. Cell Rep 23(2):637-651

9. Engler JR et al (2012) Increased microglia/macrophage gene expression in a subset of adult and pediatric astrocytomas. PLoS One 7(8):e43339

10. Figueroa ME et al (2010) Leukemic IDH1 and IDH2 mutations result in a hypermethylation phenotype, disrupt TET2 function, and impair hematopoietic differentiation. Cancer Cell 18(6):553-567

11. Fortin JP, Triche TJ Jr, Hansen KD (2017) Preprocessing, normalization and integration of the Illumina HumanMethylationEPIC array with minfi. Bioinformatics 33(4):558-560

12. Gutschner T et al (2013) The noncoding RNA MALAT1 is a critical regulator of the metastasis phenotype of lung cancer cells. Cancer Res 73(3):1180-1189

13. Heinz $S$ et al (2010) Simple combinations of lineage-determining transcription factors prime cis-regulatory elements required for macrophage and B cell identities. Mol Cell 38(4):576-589

14. Hollenhorst PC et al (2011) Oncogenic ETS proteins mimic activated RAS/ MAPK signaling in prostate cells. Genes Dev 25(20):2147-2157

15. Hothorn $T$ (2017) maxstat: Maximally Selected Rank Statistics. R package version 0.7-25. https://CRAN.R-project.org/package=maxstat.

16. Johnson BE et al (2014) Mutational analysis reveals the origin and therapydriven evolution of recurrent glioma. Science 343(6167):189-193

17. Kassambara A, Kosinski M, Biecek P (2019), survminer: Drawing Survival Curves using 'ggplot2'. R package version 0.4.5, https://CRAN.R-project.org/ package $=$ survminer 
18. Khan A et al (2018) JASPAR 2018: update of the open-access database of transcription factor binding profiles and its web framework. Nucleic Acids Res 46(D1):D260-D266

19. Labussiere M et al (2014) TERT promoter mutations in gliomas, genetic associations and clinico-pathological correlations. Br J Cancer 111(10):2024-2032

20. Langmead B, Salzberg SL (2012) Fast gapped-read alignment with bowtie 2 . Nat Methods 9(4):357-359

21. Lawrence $\mathrm{M}$ et al (2013) Software for computing and annotating genomic ranges. PLoS Comput Biol 9(8):e1003118

22. Lee SB et al (2016) An ID2-dependent mechanism for VHL inactivation in cancer. Nature 529(7585):172-177

23. Li $\mathrm{H}$ et al (2009) The sequence alignment/map format and SAMtools. Bioinformatics 25(16):2078-2079

24. Liao Y et al (2019) WebGestalt 2019: gene set analysis toolkit with revamped Uls and APIs. Nucleic Acids Res 47(W1):W199-W205

25. Ligon KL et al (2004) The oligodendroglial lineage marker OLIG2 is universally expressed in diffuse gliomas. J Neuropathol Exp Neurol 63(5): 499-509

26. Ligon KL et al (2007) Olig2-regulated lineage-restricted pathway controls replication competence in neural stem cells and malignant glioma. Neuron 53(4):503-517

27. Lu C et al (2012) IDH mutation impairs histone demethylation and results in a block to cell differentiation. Nature 483(7390):474-478

28. Lu QR et al (2002) Common developmental requirement for Olig function indicates a motor neuron/oligodendrocyte connection. Cell 109(1):75-86

29. McLean CY et al (2010) GREAT improves functional interpretation of cisregulatory regions. Nat Biotechnol 28(5):495-501

30. Morgan M et al (2019) Rsamtools: Binary alignment (BAM), FASTA, variant call (BCF), and tabix file import. $\mathrm{R}$ package version 2.0.0

31. Reon BJ et al (2018) LINC00152 promotes invasion through a 3'-hairpin structure and associates with prognosis in Glioblastoma. Mol Cancer Res 16(10):1470-1482

32. Satpathy AT et al (2019) Massively parallel single-cell chromatin landscapes of human immune cell development and intratumoral T cell exhaustion. Nat Biotechnol 37(8):925-936

33. Schep AN et al (2017) chromVAR: inferring transcription-factor-associated accessibility from single-cell epigenomic data. Nat Methods 14(10):975-978

34. Schmid T, Kruger M, Braun T (2007) NSCL-1 and -2 control the formation of precerebellar nuclei by orchestrating the migration of neuronal precursor cells. J Neurochem 102(6):2061-2072

35. Shaulian E, Karin M (2002) AP-1 as a regulator of cell life and death. Nat Cell Biol 4(5):E131-E136

36. Shrestha RK et al (2018) A workflow for simplified analysis of ATAC-cap-seq data in R. Gigascience. 7(7)

37. Suva ML et al (2014) Reconstructing and reprogramming the tumorpropagating potential of glioblastoma stem-like cells. Cell 157(3):580-594

38. Therneau TM, Grambsch PM (2000) Modeling Survival Data: Extending the Cox Model. Springer, New York

39. Tirosh I et al (2016) Single-cell RNA-seq supports a developmental hierarchy in human oligodendroglioma. Nature 539(7628):309-313

40. Turcan $\mathrm{S}$ et al (2012) IDH1 mutation is sufficient to establish the glioma hypermethylator phenotype. Nature 483(7390):479-483

41. Urbanek P et al (1997) Cooperation of Pax2 and Pax5 in midbrain and cerebellum development. Proc Natl Acad Sci U S A 94(11):5703-5708

42. van der Maaten $L$ (2014) Accelerating t-SNE using tree-based algorithms. J Mach Learn Res 15:3221-3245

43. Venteicher AS et al (2017) Decoupling genetics, lineages, and microenvironment in IDH-mutant gliomas by single-cell RNA-seq. Science. 355(6332)

44. Wang J et al (2019) HACER: an atlas of human active enhancers to interpret regulatory variants. Nucleic Acids Res 47(D1):D106-D112

45. Wang $X$ et al (2018) The long non-coding RNA CYTOR drives colorectal cancer progression by interacting with NCL and Sam68. Mol Cancer 17(1):110

46. Yan $\mathrm{H}$ et al (2009) IDH1 and IDH2 mutations in gliomas. N Engl J Med 360(8):765-773

47. Yu J et al (2019) Bioinformatics identification of IncRNA biomarkers associated with the progression of esophageal squamous cell carcinoma. Mol Med Rep 19(6):5309-5320

48. Zhang Z et al (2017) ID2 promotes survival of glioblastoma cells during metabolic stress by regulating mitochondrial function. Cell Death Dis 8(2):e2615

49. Zhao J et al (2015) Long non-coding RNA Linc00152 is involved in cell cycle arrest, apoptosis, epithelial to mesenchymal transition, cell migration and invasion in gastric cancer. Cell Cycle 14(19):3112-3123
50. Zhou Q, Anderson DJ (2002) The bHLH transcription factors OLIG2 and OLIG1 couple neuronal and glial subtype specification. Cell 109(1):61-73

51. Zhu X et al (2012) Olig2-dependent developmental fate switch of NG2 cells. Development 139(13):2299-2307

52. Zou H et al (2017) IncRNAs PVT1 and HAR1A are prognosis biomarkers and indicate therapy outcome for diffuse glioma patients. Oncotarget 8(45): $78767-78780$

\section{Publisher's Note}

Springer Nature remains neutral with regard to jurisdictional claims in published maps and institutional affiliations.
Ready to submit your research? Choose BMC and benefit from:

- fast, convenient online submission

- thorough peer review by experienced researchers in your field

- rapid publication on acceptance

- support for research data, including large and complex data types

- gold Open Access which fosters wider collaboration and increased citations

- maximum visibility for your research: over $100 \mathrm{M}$ website views per year

At BMC, research is always in progress.

Learn more biomedcentral.com/submissions 